\title{
Highly Efficient Light-Emitting Electrochemical Cells (LECs) from Host-Guest Cationic Iridium(III) Complexes*
}

\author{
Praweena Wongkaew, Usana Mahanitipong, and Natsiri Wongsang \\ Center for Organic Electronic and Alternative Energy (COEA), \\ Department of Chemistry, Faculty of Science, Ubon Ratchathani University, \\ Warinchumrap, Ubon Ratchathani Province, 34190, Thailand \\ Somboon Sahasithiwat \\ National Metal and Materials Technology Center, \\ Thailand Science Park, Pathumthani, Bangkok, 12110, Thailand \\ Rukkiat Jitchati ${ }^{\dagger}$ \\ Center for Organic Electronic and Alternative Energy (COEA), \\ Department of Chemistry, Faculty of Science, Ubon Ratchathani University, \\ Warinchumrap, Ubon Ratchathani Province, 34190, Thailand. \\ (Received 14 January 2014; Accepted 14 February 2014; Published 5 April 2014)
}

\begin{abstract}
Five charged iridium(III) complexes with a different number and position of methyl group; $\left[\operatorname{Ir}(\mathrm{phen})(\mathrm{ppy})_{2}\right] \mathrm{PF}_{6}$ (1), $\quad\left[\operatorname{Ir}(4-\right.$ phen $\left.)(\text { ppy })_{2}\right] \mathrm{PF}_{6} \quad$ (2), $\quad\left[\operatorname{Ir}(2,9-\right.$ phen $\left.)(\text { ppy })_{2}\right] \mathrm{PF}_{6} \quad(\mathbf{3}), \quad\left[\operatorname{Ir}(4,7\right.$-phen $\left.)(\mathrm{ppy})_{2}\right] \mathrm{PF}_{6} \quad(\mathbf{4}) \quad$ and $\quad[\operatorname{Ir}(4,5,6,7-$ phen)(ppy) $\left.{ }_{2}\right] \mathrm{PF}_{6}$ (5) were synthesized and characterized by NMR, IR, UV-Vis absorption, fluorescence spectroscopy and cyclic voltammetry. The host-guest light-emitting electrochemical cells (LECs) based on ITO/PEDOT:PSS/15:PVK (1:4)/ Al were investigated, which resulted in a maximum current efficiency at $1.35 \mathrm{cdA}^{-1}$ and a brightness at $717 \mathrm{cdm}^{-2}$ from 5 . The host-guest LECs can be improved by the insertion of the TPBi and LiF layers. We found that the highly efficient electroluminescence device gave the peak current efficiency to $4.49 \mathrm{cdA}^{-1}$, the maximum brightness at $1,200 \mathrm{cdm}^{-2}$ with Commission Internationale de L'Eclairage (CIE) coordinates of $(0.30,0.54)$ from 3. [DOI: $10.1380 /$ ejssnt.2014.141]
\end{abstract}

Keywords: Iridium; Nano-electronics and related devices; Nano-films; Nano-materials

\section{INTRODUCTION}

The charged iridium(III) complexes have been studied for advanced optoelectronic applications [1-4]. The advantages of these complexes are their high stabilities, high luminescence quantum yields, short excited state lifetime and tunable emission energies. Its structure compose of one $\mathrm{N}, \mathrm{N}$ bidentate and two $\mathrm{C}, \mathrm{N}$ bidentate ligands. The photophysical properties of these phosphors can be tuned by varying both $\mathrm{N}, \mathrm{N}$ and $\mathrm{C}, \mathrm{N}$ ligands [5]. Organic light-emitting diodes (OLEDs) and light-emitting electrochemical cells (LECs) have been made and studied during recent years motivated due to their potentially in display technology applications to replace LED technologies [6-8]. The charged $\operatorname{Ir}(\mathrm{III})$ complexes have been used as the emitter in both OLED and simple LEC devices [9]. Single layer LECs based on a charged iridium(III) shown by Youngson give high luminescence at 5199 and $4751 \mathrm{cdm}^{-2}$ [10]. Lian and Yong demonstrated an efficient red emitting host-guest LEC based on cationic iridium complexes. The host-guest LEC has brightness of $8.4 \mathrm{cdm}^{-2}$, external quantum efficiency (EQE) of up to $3.5 \%$ and peak current efficiency of $3.4 \mathrm{cdA}^{-1}$ [11]. Recently, Yong Qiu demonstrated a warm-white light LECs with the peak external quantum efficiency, current effi-

\footnotetext{
*This paper was presented at the 12 th International Conference on Atomically Controlled Surfaces, Interfaces and Nanostructures (ACSIN-12) in conjunction with the 21st International Colloquium on Scanning Probe Microscopy (ICSPM21), Tsukuba International Congress Center, Tsukuba, Japan, November 4-8, 2013.

${ }^{\dagger}$ Corresponding author: rukkiat_j@hotmail.com
}

ciency, and power efficiency reach $5.2 \%, 11.2 \mathrm{cdA}^{-1}$, and $10 \mathrm{lmW}^{-1}$ [12]. In this article, we show the highly efficient host-guest OLED devices based on a charged iridium(III) complex by the insertion of the TPBi and LiF layers.

\section{EXPERIMENTAL}

All chemicals were purchased from Acros organic and used without further purification, unless otherwise noted. The following five charged iridium(III) complexes (1-5) were synthesized according to the reference by varying the N,N ligands [13]. ${ }^{1} \mathrm{H}$ and ${ }^{13} \mathrm{C}$ NMR spectra were recorded on a Bruker Avance 300 spectrometer. Chemical shifts are quoted downfield from internal standard TMS. MS data were obtained using a Thermo Finnigan LTQFT instrument. Ultraviolet-visible (UV-vis) spectra were recorded with Perkin-Elmer UV Lambda 25. Photoluminescence spectrum and the fluorescence quantum yield were identified by a Perkin-Elmer LS 50B Luminescence Spectrometer the standard quinine sulfate solution in $0.01 \mathrm{M} \mathrm{H}_{2} \mathrm{SO}_{4}$, whose fluorescence quantum yield is known to be 0.54 .

(1) $\{(1,10-$ Phenanthroline-N-N' $)$-bis-(2-phenylpyridine$\left.\mathrm{C}^{2^{\prime}}, \mathrm{N}\right)$-iridium(III) $\}$ hexafluorophosphate: $(0.15 \mathrm{~g}$, $94 \%),{ }^{1} \mathrm{H}$ NMR $\left(300 \mathrm{MHz}\right.$, acetone- $\left.\mathrm{D}_{6}\right) \quad \delta 8.90$ $(\mathrm{d}, J=8.2 \mathrm{~Hz}, 1 \mathrm{H}), 8.51-8.32(\mathrm{~m}, 2 \mathrm{H}), 8.23$ $(\mathrm{d}, J=8.1 \mathrm{~Hz}, 1 \mathrm{H}), 8.07(\mathrm{dd}, J=8.2,5.0 \mathrm{~Hz}$, $1 \mathrm{H}), 7.90(\mathrm{dd}, J=15.7,7.9 \mathrm{~Hz}, 2 \mathrm{H}), 7.67(\mathrm{~d}$, $J=5.5 \mathrm{~Hz}, 1 \mathrm{H}), 7.07(\mathrm{t}, J=7.5 \mathrm{~Hz}, 1 \mathrm{H}), 6.96$ $(\mathrm{dd}, J=8.7,4.6 \mathrm{~Hz}, 2 \mathrm{H}), 6.45(\mathrm{~d}, J=7.4 \mathrm{~Hz}$, $1 \mathrm{H}) ;{ }^{13} \mathrm{C}$ NMR $\left(75 \mathrm{MHz}\right.$, acetone- $\left.\mathrm{D}_{6}\right) \delta 167.81$, $151.27,149.87,149.37,146.93,144.29,138.75$, 
$138.55, \quad 131.79, \quad 131.71, \quad 130.32, \quad 128.45, \quad 126.99$, 124.90, 123.43, 122.56, 119.81; Ms $\left(\mathrm{ES}^{+}\right): \mathrm{m} / \mathrm{z}(\%)$ $681.1630\left(\mathrm{M}^{+}-\mathrm{PF}_{6}, 100\right)$. The characterizations are also reported elsewhere [13-15].

(2) \{(4-Methyl-1,10-phenanthroline-N-N')-bis- (2phenylpyridine- $\left.\mathrm{C}^{2^{\prime}}, \mathrm{N}\right)$-iridium(III) $\}$ hexafluorophosphate: (0.08 g, 49\%), ${ }^{1} \mathrm{H}$ NMR (300 MHz, acetone- $\left.\mathrm{D}_{6}\right) \delta 8.86(\mathrm{~d}, J=8.2 \mathrm{~Hz}, 1 \mathrm{H}), 8.50(\mathrm{~d}$, $J=9.1 \mathrm{~Hz}, 1 \mathrm{H}), 8.40(\mathrm{t}, J=7.4 \mathrm{~Hz}, 2 \mathrm{H}), 8.23$ $(\mathrm{dd}, J=13.2,6.7 \mathrm{~Hz}, 3 \mathrm{H}), 8.01(\mathrm{dd}, J=8.2$, $5.1 \mathrm{~Hz}, 1 \mathrm{H}), 7.88(\mathrm{dd}, J=14.3,7.1 \mathrm{~Hz}, 5 \mathrm{H})$, $7.66(\mathrm{~d}, J=5.6 \mathrm{~Hz}, 2 \mathrm{H}), 6.99$ (ddd, $J=18.5$, $14.8,7.3 \mathrm{~Hz}, 6 \mathrm{H}), 6.54-6.36(\mathrm{~m}, 2 \mathrm{H}), 2.99(\mathrm{~s}, 3 \mathrm{H})$; ${ }^{13} \mathrm{C} \mathrm{NMR}\left(75 \mathrm{MHz}, \mathrm{CDCl}_{3}\right) \delta 205.44,167.84$, $167.80,151.25,150.44,150.27,149.27,149.23$, $147.03,146.50,144.35,144.28,138.64,138.52$, $131.84,131.79,131.46,131.30,130.29,128.09$, $127.52,126.85,125.09,124.89,123.46,123.44$, 122.47, 119.80, 18.22; Ms $\left(\mathrm{ES}^{+}\right): \mathrm{m} / \mathrm{z}(\%) 740.1618$ $\left(\mathrm{M}^{+}-\mathrm{PF}_{6}+2 \mathrm{Na}^{+}, 100\right)$.

(3) \{(2,9-Dimethyl-1,10-phenanthroline-N-N' $\left.{ }^{\prime}\right)$-bis- $(2-$ phenylpyridine- $\left.\mathrm{C}^{2^{\prime}}, \mathrm{N}\right)$-iridium(III) $\}$ hexafluorophosphate: (0.145 g, 91\%), ${ }^{1} \mathrm{H}$ NMR (300 MHz, $\left.\mathrm{CDCl}_{3}\right) \delta 8.72(\mathrm{~d}, J=8.3 \mathrm{~Hz}, 1 \mathrm{H}), 8.23(\mathrm{t}$, $J=3.7 \mathrm{~Hz}, 2 \mathrm{H}), 7.92(\mathrm{t}, J=7.8 \mathrm{~Hz}, 1 \mathrm{H}), 7.82(\mathrm{t}$, $J=6.9 \mathrm{~Hz}, 3 \mathrm{H}), 7.01(\mathrm{t}, J=6.6 \mathrm{~Hz}, 1 \mathrm{H}), 6.95(\mathrm{t}$, $J=7.6 \mathrm{~Hz}, 1 \mathrm{H}), 6.79(\mathrm{t}, J=7.4 \mathrm{~Hz}, 1 \mathrm{H}), 6.21(\mathrm{~d}$, $J=7.6 \mathrm{~Hz}, 1 \mathrm{H}), 2.21(\mathrm{~s}, 3 \mathrm{H}) ;{ }^{13} \mathrm{C} \mathrm{NMR}(75 \mathrm{MHz}$, Acetone- $\left.\mathrm{D}_{6}\right) \quad \delta$ 205.34, 205.07, 167.85, 165.19, $150.15,148.95,148.00,143.48,139.22,138.50$, $131.57,130.04,129.71,128.03,127.24,124.82$, 123.04, 122.10, 119.80, 26.8; $\mathrm{Ms}\left(\mathrm{ES}^{+}\right): \mathrm{m} / \mathrm{z}(\%)$ $709.1943\left(\mathrm{M}^{+}-\mathrm{PF}_{6}, 100\right)$. The characterizations are also reported elsewhere [16].

(4) \{(4,7-Dimethyl-1,10-phenanthroline-N-N')-bis- (2phenylpyridine- $\left.\mathrm{C}^{2^{\prime}}, \mathrm{N}\right)$-iridium(III) $\}$ hexafluorophosphate: (0.15 g, 94\%), ${ }^{1} \mathrm{H}$ NMR (300 MHz, $\left.\mathrm{CDCl}_{3}\right) \delta 8.24(\mathrm{~s}, 1 \mathrm{H}), 8.08(\mathrm{~d}, J=5.1 \mathrm{~Hz}, 1 \mathrm{H})$, $7.87(\mathrm{~d}, J=8.1 \mathrm{~Hz}, 1 \mathrm{H}), 7.74-7.58(\mathrm{~m}, 2 \mathrm{H}), 7.52$ $(\mathrm{d}, J=5.1 \mathrm{~Hz}, 1 \mathrm{H}), 7.34(\mathrm{~d}, J=5.5 \mathrm{~Hz}, 1 \mathrm{H})$, 7.04-6.79 (m, 3H), 6.39 (d, J = 7.3 Hz, 1H), 2.85 $(\mathrm{s}, 3 \mathrm{H}) ;{ }^{13} \mathrm{C} \mathrm{NMR}\left(75 \mathrm{MHz}, \mathrm{CDCl}_{3}\right) \delta 167.72$, $150.33,150.16,148.94,148.56,146.45,143.85$, $138.16,131.93,131.03,130.62,127.23,124.73$, 123.40, 122.53, 119.58, 19.04; Ms $\left(\mathrm{ES}^{+}\right): \mathrm{m} / \mathrm{z}(\%)$ $709.1943\left(\mathrm{M}^{+}-\mathrm{PF}_{6}, 100\right)$. The characterizations are also reported elsewhere [17].

(5) $\{(3,4,7,8$-Tetramethyl-1,10-phenanthroline-N$\mathrm{N}^{\prime}$ )-bis-(2-phenylpyridine- $\left.\mathrm{C}^{2^{\prime}}, \mathrm{N}\right)$-iridium(III) $\}$ hexafluorophosphate: $(0.15 \mathrm{~g}, 92 \%) ;{ }^{1} \mathrm{H}$ NMR $\left(300 \mathrm{MHz}, \mathrm{CDCl}_{3}\right) \delta 8.26(\mathrm{~s}, 1 \mathrm{H}), 7.91(\mathrm{~s}, 1 \mathrm{H})$, $7.89(\mathrm{~d}, J=8.3 \mathrm{~Hz}, 1 \mathrm{H}), 7.68(\mathrm{t}, J=6.9 \mathrm{~Hz}, 2 \mathrm{H})$, $7.36(\mathrm{~d}, J=5.6 \mathrm{~Hz}, 1 \mathrm{H}), 7.02(\mathrm{t}, J=7.1 \mathrm{~Hz}$, $1 \mathrm{H}), 6.92(\mathrm{dd}, J=10.6,6.1 \mathrm{~Hz}, 2 \mathrm{H}), 6.38(\mathrm{~d}$, $J=7.3 \mathrm{~Hz}, 1 \mathrm{H}), 2.78(\mathrm{~s}, 3 \mathrm{H}), 2.36(\mathrm{~s}, 3 \mathrm{H}) ;{ }^{13} \mathrm{C}$ $\mathrm{NMR}\left(75 \mathrm{MHz}, \mathrm{CDCl}_{3}\right) \delta 167.80,151.03,150.60$, $148.70,146.38,145.41,143.74,137.88,135.06$, $131.88,130.67,130.02,124.63,124.42,123.31$, $122.42,119.41,18.07,14.99 ; \mathrm{Ms}\left(\mathrm{ES}^{+}\right): \mathrm{m} / \mathrm{z}(\%)$

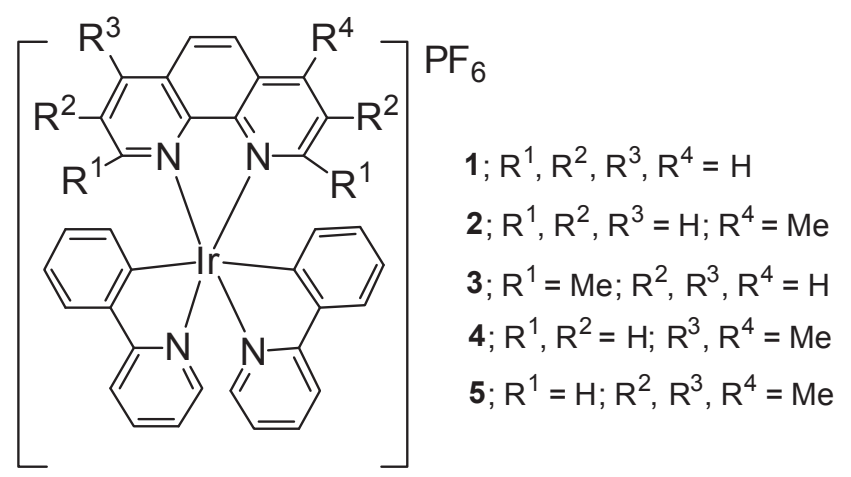

FIG. 1: The molecular structure of complex 1-5.

$737.2256\left(\mathrm{M}^{+}-\mathrm{PF}_{6}, 100\right)$. The characterizations are also reported elsewhere [15].

The electroluminescence was fabricated with a solution of the iridium complexes in acetonitrile with a concentration of $12.5 \mathrm{mg} \mathrm{ml}^{-1}$. Prior to the fabrication, the ITO glass substrates were thoroughly cleaned, followed by UVozone treatment. A thin film of PEDOT:PSS layer was spin coated at $3000 \mathrm{rpm}$ for $180 \mathrm{~s}$ and baked at $260^{\circ} \mathrm{C}$ for 10 min. For simple LEC device, the complex 1-5 film was spin-coated with poly(9-vinylcarbazole) (PVK) as a host at 1:4 mole ratio, followed by the aluminum cathode using a shadow mask under high vacuum. For the OLED device, 1,3,5-tris (2-N-phenylbenzimidazolyl)benzene (TPBI) and $\mathrm{LiF}$ were used before aluminum cathode. The electroluminescent properties of the resultant devices were performed using Keithley characterization systems. The current density and luminance versus voltage sweeps were measured using a Keithley 2400 source meter and calibrated with a silicon photodiode.

\section{RESULTS AND DISCUSSION}

The molecular structure of complex 1-5 is depicted in Fig. 1. The charged iridium(III) complexes were designed with 2-phenyl pyridine as the C,N ligand and 1,10phenanthroline as the $\mathrm{N}, \mathrm{N}^{\prime}$ ligand by varying the number and the position of methyl groups.

The solution absorption and emission of complex 1$\mathbf{5}$ were shown in Fig. 2. The strong absorption bands below $300 \mathrm{~nm}$ can be assigned as the spin-allowed $\pi-\pi^{*}$ transition in the ligand-centered (LC) transition of the cyclometalated phenyl pyridine (ppy) and phenanthroline (phen) while the weak absorption region shows at 385$500 \mathrm{~nm}$ corresponding to metal-to-ligand charge transfer (MLCT) transition, respectively. These characteristic are consistent with the previously reported of the literature data [14-17]. The emission wavelength of the complex 1$\mathbf{5}$ shows at $518-547 \mathrm{~nm}$ with the emission quantum yield about 0.01-0.02 using a solution of quinine sulfate served as the standard. We found that the photo physic properties of the solution and thin film data are slightly red shifted. These characteristic indicated that the aggregate was formed in solid thin film. The photophysic data are summarized in Table I. 
TABLE I: Photophysical characteristics of solution and thin film from complex 1-5.

\begin{tabular}{|c|c|c|c|c|c|}
\hline \multirow[t]{2}{*}{ Complex } & \multicolumn{3}{|c|}{ DCM solution } & \multicolumn{2}{|c|}{ Thin film $^{\mathrm{a}}$} \\
\hline & $\lambda_{\text {abs }}(\mathrm{nm}, \log \varepsilon)$ & $\lambda_{\mathrm{em}}(\mathrm{nm})$ & $\Phi_{\mathrm{PL}}$ & $\lambda_{\text {abs }}(\mathrm{nm})$ & $\lambda_{\mathrm{em}}(\mathrm{nm})$ \\
\hline 1 & $267(4.9), 381(4.0), 418(3.7)$ & 547 & 0.01 & 385,421 & 551 \\
\hline 2 & $267(4.7), 381(3.7), 414(3.4)$ & 540 & 0.01 & 381,424 & 545 \\
\hline 3 & $271(4.3), 375(3.2), 419(2.7)$ & 524 & 0.02 & 420 & 536 \\
\hline 4 & $268(4.7), 381(3.8), 416(3.5)$ & 535 & 0.01 & 418 & 540 \\
\hline 5 & $272(4.5), 343(3.8), 421(3.2)$ & 518 & 0.01 & 424 & 538 \\
\hline
\end{tabular}

${ }^{\mathrm{a}}$ Spin-coated from DCM solution.

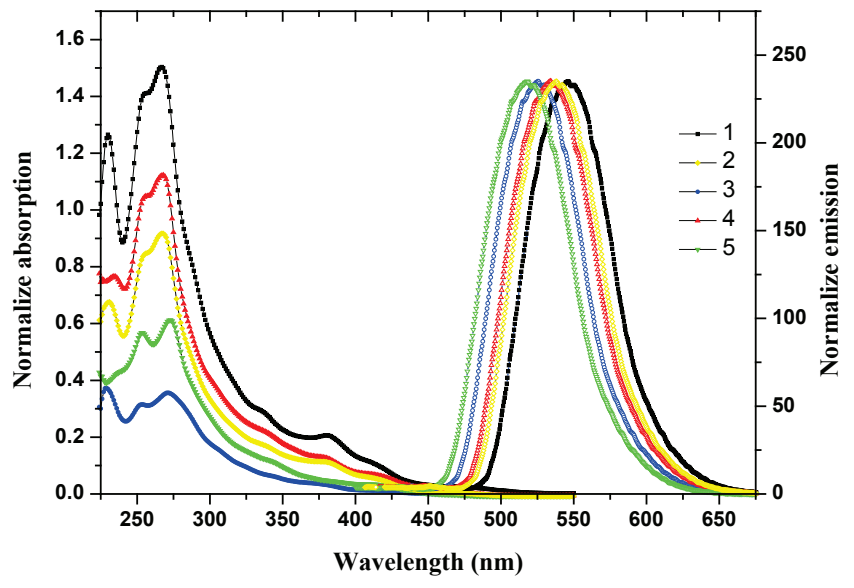

FIG. 2: The UV-Visible absorption and emission of complex 1-5.

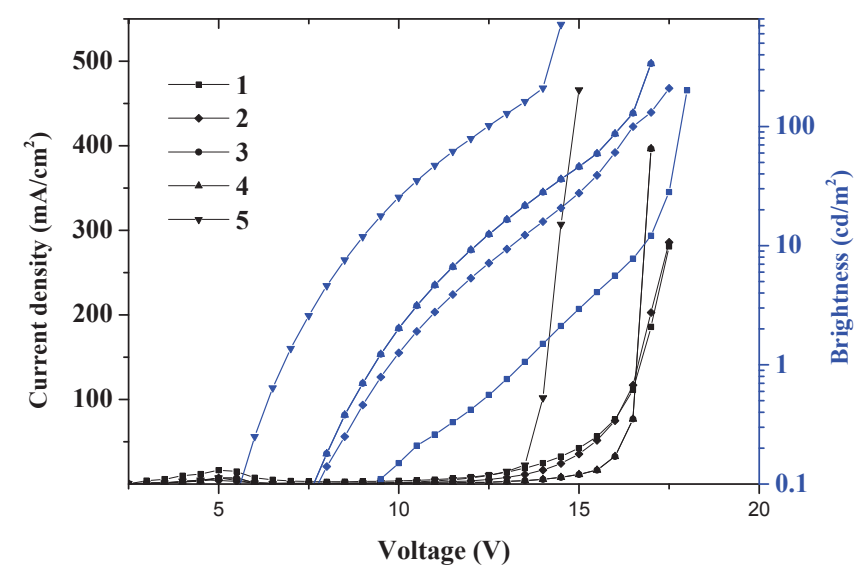

FIG. 3: Current density and brightness versus applied bias voltage of the device structure ITO/PEDOT:PSS /PVK: 1-5 $(4: 1) / \mathrm{Al}$.

The electroluminescences of the complex 1-5 were investigated with a simple LECs by using 4:1 host-guest ratio as in our previous study [18]. We fabricated the LEC device with the structure: ITO/PEDOT:PSS/PVK: 1-5 (4:1)/Al which PEDOT:PSS were used as a hole-injecting layer and PVK as the high triplet energy host polymer. We found that our host-guest LECs of complex $\mathbf{1}$ give significantly increase a brightness to $201 \mathrm{cdm}^{-2}$ compared with a pure LECs device at $40 \mathrm{cdm}^{-2}$ [14]. The results show that a maximum current efficiency at $1.35 \mathrm{cdA}^{-1}$ at

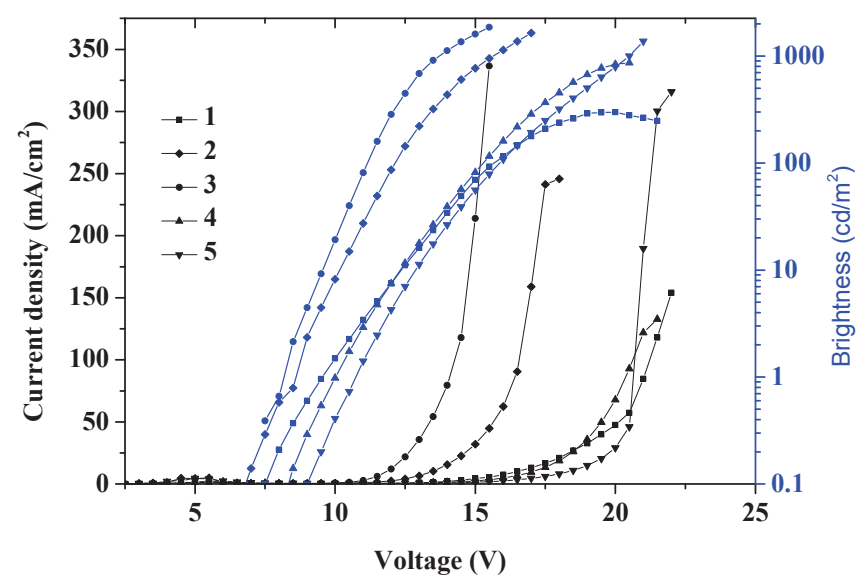

FIG. 4: Current density and brightness versus applied bias voltage of the device structure ITO/PEDOT:PSS /PVK: 1-5 (4:1)/TPBI/LiF/Al.

TABLE II: Host-guest LEC and OLED device performances of complex 1-5.

\begin{tabular}{cccccc}
\hline \hline complex & $\begin{array}{c}V_{\text {turn-on }} \\
(\mathrm{V})\end{array}$ & $\begin{array}{c}L_{\max } \\
\left(\mathrm{cdm}^{-2}\right)\end{array}$ & $\begin{array}{c}\eta_{\mathrm{c}} \\
\left(\mathrm{cdA}^{-1}\right)\end{array}$ & $\begin{array}{c}\eta_{\mathrm{p}} \\
\left(\mathrm{lmW}^{-1}\right)\end{array}$ & $\begin{array}{c}\text { CIE } \\
(x, y)\end{array}$ \\
\hline $\mathbf{1}^{\mathrm{a}}$ & 13.4 & 201 & 0.13 & 0.02 & $0.46,0.63$ \\
$\mathbf{2}^{\mathrm{a}}$ & 9.8 & 209 & 0.14 & 0.04 & $0.41,0.53$ \\
$\mathbf{3}^{\mathrm{a}}$ & 9.3 & 337 & 0.55 & 0.14 & $0.39,0.58$ \\
$\mathbf{4}^{\mathrm{a}}$ & 9.3 & 393 & 0.55 & 0.14 & $0.40,0.59$ \\
$\mathbf{5}^{\mathrm{a}}$ & 6.8 & 717 & 1.35 & 0.42 & $0.31,0.55$ \\
$\mathbf{1}^{\mathrm{b}}$ & 13.4 & 299 & 1.67 & 0.38 & $0.33,0.49$ \\
$\mathbf{2}^{\mathrm{b}}$ & 9.8 & 1,642 & 3.49 & 0.89 & $0.30,0.50$ \\
$\mathbf{3}^{\mathrm{b}}$ & 9.3 & 1,200 & 4.49 & 1.14 & $0.30,0.54$ \\
$\mathbf{4}^{\mathrm{b}}$ & 9.3 & 1,859 & 2.90 & 0.83 & $0.26,0.47$ \\
$\mathbf{5}^{\mathrm{b}}$ & 6.8 & 1,376 & 4.28 & 0.84 & $0.30,0.52$ \\
\hline
\end{tabular}

a Device 1: ITO/PEDOT:PSS/PVK: 1-5 (4:1)/Al

${ }^{\mathrm{b}}$ Device 2: ITO/PEDOT:PSS/PVK: 1-5 (4:1)/TPBI/LiF/Al

the brightness $710 \mathrm{cdm}^{-2}$ can be observed with complex 5 (Fig. 3).

To further study the host-guest EL properties, the complex 1-5 were fabricated with OLED structure: ITO/PEDOT:PSS/PVK: 1-5 (4:1)/TPBI/LiF/Al. The device inserted $\mathrm{TBBI} / \mathrm{LiF}$ as the electron transporting layers. All OLEDs gave more efficient electroluminescence than simple LEC devices which can be explained by the energy profile $[9,19]$. The best device can be achieved from complex 3 with a current efficiency of $4.49 \mathrm{cdA}^{-1}$, maximum brightness at $1,200 \mathrm{cdm}^{-2}$, turn on voltage of $9.3 \mathrm{~V}$ and CIE coordinates at $0.30,0.54$ (Fig. 4). All the 
electrical characteristics are summarized in Table II.

\section{CONCLUSIONS}

The series of charged $\operatorname{Ir}(\mathrm{III})$ complexes with 1,10phenanthroline as N,N ligand have been synthesized and fabricated with host-guest electroluminescence. Our devices demonstrated that OLEDs give a better efficient than LECs device. The results show that ITO/PEDOT:PSS/PVK: 3 (4:1)/TPBI/LiF/Al give a maximum current efficiency at $4.49 \mathrm{cdA}^{-1}$ and maximum brightness at $1,200 \mathrm{cdm}^{-2}$.

\section{Acknowledgments}

This work was financially supported by the Thailand Research Fund, the Higher Education Commission and Ubon Ratchathani University (MRG5480005). Usana Mahanitipong thanks to the Young Scientist and Technologist Program (YSTP) for the scholarship.
[1] J. Slinker, D. Bernards, P. L. Houston, H. D. Abruna, S. Bernhard, and G. G. Malliaras, Chem. Commun. 19, 2392 (2003).

[2] J. D. Slinker, J. Rivnay, J. S. Moskowitz, J. B. Parker, S. Bernhard, H. D. Abruna, and G. G. Malliaras, J. Mater. Chem. 17, 2976 (2007).

[3] R. C. Evans, P. Douglas, and C. J. Winscom, Coord. Chem. Rev. 250, 2093 (2006).

[4] G.-G. Shan, D.-X. Zhu, H.-B. Li, P. Li, Z.-M. Su, and Y. Liao, Dalton Trans. 40, 2947 (2011).

[5] L. He, L. Duan, J. Qiao, G. Dong, L. Wang, and Y. Qiu, Chem. Mater. 22, 3535 (2010).

[6] H.-B. Wu, H.-F. Chen, C.-T. Liao, H.-C. Su, and K.-T. Wong, Org. Electron. 13, 483 (2012).

[7] H. A. Al-Attar, G. C. Griffiths, T. N. Moore, M. Tavasli, M. A. Fox, M. R. Bryce, and A. P. Monkman, Adv. Funct. Mater. 21, 2376 (2011).

[8] R. D. Costa, O. Enrique, H. J. Bolink, S. Graber, C. E. Housecroft, and E. C. Constable, Adv. Funct. Mater. 20, 1511 (2010).

[9] L. He, L. Duan, J. Qiao, D. Zhang, G. Dong, L. Wang, and Y. Qiu, Synth. Met. 166, 52 (2013).

[10] C. D. Sunesh, M. Chandran, G. Mathai, and Y. Choe, Opt. Mater. 35, 407 (2013).
[11] T. Hu, L. Duan, J. Qiao, L. He, D. Zhang, L. Wang, and Y. Qiu, Synth. Met. 163, 33 (2013).

[12] L. He, J. Qiao, L. Duan, G. Dong, D. Zhang, L. Wang, and Y. Qiu, Adv. Funct. Mater. 19, 2950 (2009).

[13] C. Rothe, C.-J. Chiang, V. Jankus, K. Abdullah, X. Zeng, R. Jitchati, A. S. Batsanov, M. R. Bryce, and A. P. Monkman, Adv. Funct. Mater. 19, 2038 (2009).

[14] R. D. Costa, E. Orti, H. J. Bolink, S. Graber, S. Schaffner, M. Neuburger, C. E. Housecroft, and E. C. Constable, Adv. Funct. Mater. 19, 3456 (2009).

[15] M. S. Lowry, W. R. Hudson, R. A. Pascal, and S. Bernhard, J. Am. Chem. Soc. 126, 14129 (2004).

[16] D. B. Moon and Y. S. Choe, Mol. Cryst. Liq. Cryst. 584, $60(2013)$

[17] C. Dragonetti, L. Falciola, P. Mussini, S. Righetto, D. Roberto, R. Ugo, A. Valore, F. DeAngelis, S. Fantacci, A. Sgamellotti, M. Ramon, and M. Muccini, Inorg. Chem. 46, 8533 (2007).

[18] P. Khamtoem, S. Sahasithiwat, M. A. Fox and R. Jitchati, e-J. Surf. Sci. Nanotech. (Submitted).

[19] F. Dumur, D. Bertin, C. R. Mayer, A. Guerlin, G. Wantz, G. Nasr, E. Dumas, F. Miomandre, G. Clavier, and D. Gigmes, Synth. Met. 161, 1934 (2011). 